\title{
Foresights of Failure: An Appreciation of Barry Turner
}

\author{
Karl E. Weick*
}

One of Barry Turner's heroes was Tom Burns, co-author (with Stalker) of the (1961) book The Management of Innovation. What Turner liked about Burns' style of scholarship was his use of over-lapping accounts to grasp the subject matter, a style that Turner described as much like 'shaking a kaleidoscope' (1995: 283). That imagery is telling because it suggests that Turner, in his own writing, may be doing something that is quite different from the run-ofthe-mill scholar who provides a 'new lens' to grasp phenomena. This paper seeks to argue that the staying power of Man-Made Disasters is due, in part, to the fact that it is not a lens, but rather a kaleidoscope.

The contrast between a lens and kaleidoscope comes from Nord and Connell (1993), who point out that the lens metaphor originated with Kuhn (1970) and was his way of illustrating how scientists, guided by different paradigms, see quite different patterns in the same subject matter. What is interesting is that the metaphor of a lens assumes a realist position, the lens sizes up something out there. As Nord and Connell (1993: 117) put it:

Just as the image of switching lenses can represent
the changing of patterns in the realist schema, the
image of turning a kaleidoscope can represent the
changing of patterns in the subjectivist schema,
since the patterns of a kaleidoscope may be
internally generated with minimal dependence on
information from outside. Turning a kaleidoscope
can: (1) dislodge old patterns, (2) generate new
patterns and (3) foster awareness that numerous
configurations are possible.

These images come together in Burrell and Morgan's (1979) description of Turner as an '... ontologically confused social realist', a label that Turner (1995: 282) says he '.. always took more as a perceptive description than as a fault needing correction'. The label is perceptive in the sense that Turner (1995: 281) reports himself to be 'sympathetic to situational accounts of the world, where meaning and significance are provisional and socially constructed'. What is even more perceptive in that label is that it captures the human pre-conditions to disaster that Turner went on to articulate. Disasters often seem to be the work of ontologically confused social realists in organizations who are beset with perceptual rigidities, information ambiguities, dis-regard of rules, susceptibility to decoys, over-confidence, hubris and mis-placed concreteness. While Turner excelled as an observer, what becomes clear in re-reading Man-Made Disasters is that he also had mastery of pattern generation with sufficient requisite variety to match and register the patterned variety in the complex events he studied. It is this mastery of pattern creation that is best captured by the imagery of a kaleidoscope.

This author wants to approach the re-issue of Turner's book appreciatively by showing how it continues to influence our current thinking about the social organization of mistakes in a kaleidoscope-like fashion. The work dis-lodges old patterns, generates new patterns and fosters awareness that numerous configurations are possible in the genesis of disasters.

As an example of dis-lodging old patterns, consider Turner's (1978) discussion of order as a two-edged sword. To organize is to make relationships more orderly, more predictable, more dependable, more consistent and to reverse centrifugal tendencies toward entropy, dis-order and fractionation. Obvious as all of this may be, what theorists repeatedly miss is the fact that an increase in order increases both the likelihood that tasks will be accomplished as intended and the likelihood that mistakes or anti-tasks, as Turner calls them, will also be accomplished and diffuse more widely. 'The more extensive a negentropic order-seeking system becomes, the greater is the potential which it also develops for the orderly dissemination of unintended consequences' (Turner, 1978: 180). Any organized hierarchy can amplify errors, mis-information or mis-understanding that occur early in a performance sequence or near the top of a hierarchy. Thus, one can argue that organizations execute both tasks and anti-tasks and as they accomplish ness School, 701 Tappan Street Ann Arbor, MI 48109-1234, United States. 
both of them more successfully, the better organized they are.

Several old patterns are dis-lodged by Turner's (1978) argument that increased order facilitates the performance of tasks and antitasks. It may be that the very routineness, order and reliability that High Reliability Organizations (HROs) cultivate, encourages small errors to spread and be seeded in numerous places. This dispersion increases the probability that separate errors will become aligned inter-actively and trigger serious consequences. Thus, HROs may be failure-free in spite of their order-seeking, not because of it. If this is plausible, then, it could provide an answer to the puzzle of how HROs can simultaneously be relatively failure-free and yet seem to resemble 'garbage cans' rather than mechanistic systems. A common presumption in Organization Theory is that 'systems with catastrophic potential are particularly vulnerable to the problems of bounded rationality and 'Garbage-Can' processes which will encourage multiple errors and de-emphasize safety goals' (Perrow, 1994: 219). The less common presumption, one being explored in recent work on mindfulness in high reliability organizing (Weick, Sutcliffe and Obstfeld, 1997), would be that garbage-can systems have fewer pathways for anti-tasks and, therefore, are safer. It is the moderation of order, not the expansion of order, that lies behind failure-free performance. Furthermore, garbage-can structures tend to be flat structures. This property becomes important in the context of Turner's argument that the higher in a hierarchy an error occurs and the more negentropic the organization, the more that error should be magnified, the more likely it is that the error will be compounded with other errors and the more likely it is that the error will be disastrous (Turner, 1978: 187).

Turner's discussion of order also helps us dislodge thinking about bureaucracy. It helps us, for example, re-think La Porte and Consolini's (1991: 31) observation that most HROs exhibit a:

bureaucratic mode of operations much of the time. This forms the ordering, status/rank-oriented background structure of the organization and is adequate for organizational responses to low to moderate demand. Is this structure adequate for response during peakload or high-tempo operations? ... Peak demands or high-tempo activities become a solvent of bureaucratic forms.

What interests us is the possibility, not yet examined, that it is this very same bureaucratic mode of operation that allows small errors to accumulate, thereby increasing the need for eventual high-tempo moments to deal with them. Problems gain headway during normal bureaucratic times when tasks and anti-tasks coevolve. By the time a high-tempo structure replaces a bureaucratic structure, the 'damage' has already been done. Errors have been allowed to unfold and to interact in an orderly manner with other failures. Normal operations give way to high-tempo operations, not so much because demands have changed, but because normal operations have allowed failures to accumulate and grow to dangerous levels. Dependencies between normal- and high-tempo operations may be higher than previously imagined, if we take Turner seriously. And, if this is true for HROs, it may also be true for organizations in general.

Thus, failure comes from either a loss of order, something always suspected, or from an increase in order, a newer suspicion that is consistent with what is known about phenomena such as vicious circles, escalation and anti-tasks. Effective organizations are vulnerable to failure if their success derives from tight coupling, integration or other practices of organizing that make it easier for small, high-failures to spread. If, however, effectiveness is accomplished through loosened coupling, less unitary designs, pluralism and federations, then pathways for errors should be fewer and shorter. If these conjectures are plausible, then they suggest that so-called 'safety cultures' work, not because they are strong cultures (strong cultures are unitary), but because they are weaker cultures which give general guidance. Strong cultures can compromize safety if they provide strong social order that encourages the compounding of small failures.

The second property of kaleidoscopes, their capacity to generate new patterns, can be illustrated by Turner's discussion of variable disjunction. He argues that it is common for disasters to happen 'when a large complex problem, the limits of which were difficult to specify, was being dealt with by a number of groups and individuals usually operating in separate organizations' (Turner, 1976: 384). Problems of this kind were said to have information that was variably disjunctive. This term refers 'to a complex situation in which a number of parties handling a problem are unable to obtain precisely the same information about the problem so that many differing interpretations of the problem exist' (Turner, 1978 :50). What makes this condition so relevant to all contemporary organizations is that it is likely to be found 'when problems use symbolic or verbal variables, have vague non-quantifiable goals and lack available routines for their solution, relying instead on ad hoc procedures, a variable disjunction of information is likely to be found' (Turner, 1978: 52).

Notice that variable disjunction, which is a problem of social structure, can cause confusion and incomprehension much like the confusion 
caused by technological puzzles in the form of inter-active complexity (Perrow, 1984). Though the origins are quite different, the outcomes are the same. Thus, problems that produce disasters can ramify in unexpected ways, not because technological events are incomprehensible but because dispersed people have diverse, nonoverlapping pieces of information about what is happening and different interpretations as a result. Each person has partial information that is incomprehensible because crucial pieces are missing. Technology is less of a problem than is the way people are organized. Given the tendency of people to satisfice and make do with what information they have, it is possible that accidents happen because information is incomplete but the gaps are smoothed over in ways that sustain the illusion of safety.

But it is also possible that accidents happen when attempts are made to assemble disjunctions. When people attempt to reconcile separate, selfcontained, non-overlapping interpretations, these efforts may make sense to no one, which again represents a social origin of incomprehension. Those who persist in their efforts to comprehend, may ignore most of what is distinctive and diagnostic and focus on those features on which everyone agrees. Too often, these common interpretations are the very ones that create a joint blind-spot. This is precisely what happened in the Challenger disaster. When disjunct information was assembled around the common interpretation that the O-rings were redundant, observers missed the culturally alien datum that the lower temperatures at launch were associated with a greater incidence of O-ring failure. Disjunctions were reconciled by omitting the very information they were meant to catch.

The final property of a kaleidoscope, its ability to foster an awareness that numerous configurations are possible, is illustrated by the deceptively simple manner in which Turner (1978) begins his argument. In order to act collectively, people adopt simplifying assumptions. Simplifications limit the precautions people take and the range of undesired consequences they envision. These simplifications set the stage for surprise. They allow anomalies to accumulate, assumptions to be dis-confirmed and un-desired consequences to grow more serious (all of these could qualify as ontological confusions). Thus, from the perspective of research on organizations with catastrophic potential, perhaps the most crucial fact about these organizations is that they achieve a minimal level of coordination by persuading their decisionmakers to agree that they will all neglect the same kind of consideration when they make decisions' (Turner, 1978: 166). Organizations are defined by what they ignore - ignorance that is embodied in assumptions - and by the extent to which people in them neglect the same kinds of considerations.
Thus, members of organizations solve the problem of what to do next by simplifying the manner in which the current situation is interpreted. These simplifications, variously referred to as world-views, frameworks or, more globally, as culture, basically tell members what they can afford to ignore in order to reduce their confusion. The success of these simplifications, however:

... turns on the issue of whether the simplified diagnosis of the present and likely future situation is accurate enough, to enable the organizational goals to be achieved without encountering unexpected difficulties that lead on to catastrophe. The central difficulty, therefore, lies in discovering which aspects of the current set of problems facing an organization are prudent to ignore and which should be attended to, and how an acceptable level of safety can be established as a criterion in carrying out this exercise (Turner, 1976: 379).

In this analysis, it is assumptions, not routines, that carry an organization's learning, as well as its blind-spots. Furthermore, it is not so much the magnitude of consequences that is crucial to understand organizations with the potential for catastrophe, as it is the character of their assumptions. Assumptions conceal warning signals, deflect attention to safe issues, leave signals unnoticed because they are undefined and set the stage for surprises that necessitate revision in administrative practices.

When one focuses on assumptions, one sees continuities across organizations, catastrophes no longer seem so exotic and all organizations appear more vulnerable than they admit. The basic continuities in Turner's (1978) analysis are:

- All organizations develop culturally accepted beliefs about the world and its hazards;

- all organizations develop associated precautionary norms set out in laws, codes, rules; and

- all organizations accumulate unnoticed events that are at odds with accepted beliefs about hazards and norms for avoiding those hazards.

Intentions, assumptions and beliefs about the world and its hazards are found in any collective activity. Attention is colored by intentions and that is why assumptions and issues of reliability are wedded so closely together. Since there is so much information in HROs, and since so much of that information is ambiguous, there is a need for structure of some kind. Since much of that structure comes from assumptions and what people choose to take for granted and to ignore, world-views are important in all organizations that face similar complexities. Disturbing as it may seem when the stakes are this high (Perrow, 1994), organizations seem to operate as if believing is seeing. They live by institutional 
scripts that act like paradigms which exclude a lot, yet survive when challenged (Vaughan, 1996).

As should be clear, Turner's work is more important now than ever given a changing world of global organizations where it should be increasingly easy to trigger unintended consequences. A changing world is, after all, a fuzzy world in which deviations from intentions will be tough to spot, information is spread around and variably disjunctive, everything feels like an anomaly and mistakes will be indigenous to whatever work seems appropriate. Given this configuration, one would presume it to be commonplace for 'unnoticed, mis-perceived, and mis-understood events to accumulate in a manner that leads to cultural disruption' (Turner, 1976: 382).

A world patterned with that level of complexity takes more than a lens to understand it. It takes a complex guide, comfortable with his confusion, to spot the confusion in others and what it leads to. And it takes a kaleidoscopic sensitivity to render clearly the multiple patterns that eventuate in disaster. This re-issue of ManMade Disasters reminds us that Barry Turner is one of the best minds ever to have tackled this nest of issues.

\section{References}

Burns, T. and Stalker (1961), The Management of
Innovation, Tavistock, London.

Burrell, G. and Morgan, G. (1979), Sociological Paradigms and Organizational Analysis, Heinemann, London.

Kuhn, T.S. (1970), The Structure of Scientific Revolutions, University of Chicago Press, Chicago.

LaPorte, T. and Consolini, P.M. (1991), 'Working in Practice But Not in Theory', Journal of Public Administration Research and Theory, Volume 1, Number 1, Winter, pp. 19-47.

Nord, W.R. and Connell, A.F. (1993), 'From Quicksand to Crossroads: An Agnostic Perspective on Conversation', Organization Science, Volume 4, Number 1, February, pp. 108-120.

Perrow, C. (1984), Normal Accidents: Living with Highrisk Technologies, Basic Books, New York.

Perrow, C. (1994), 'The Limits of Safety: The Enhancement of a Theory of Accidents', Journal of Contingencies and Crisis Management, Volume 2, Number 4, December, pp. 212-220.

Turner, B. (1976), 'The Organizational and Interorganizational Development of Disasters', Administrative Science Quarterly, Volume 21, Number 3, September, pp. 378-397.

Turner, B. (1978), Man-Made Disasters, Wykeham Publications, London.

Turner, B. (1995), 'A Personal Trajectory Through Organization Studies', Research in the Sociology of Organizations, Volume 13, pp. 275-301.

Vaughan, D. (1996), The Challenger Launch Decision: Risky Technology, Culture and Deviance at NASA, University of Chicago Press, Chicago.

Weick, K.E., Sutcliffe, K.M. and Obstfeld, D. (1997), Organizing for High Reliability: Resilience Through Collective Mindfulness, University of Michigan (Unpublished Manuscript). 